\title{
Who engaged in home-based arts activities during the COVID-19 pandemic? A cross-sectional analysis of data from 4,731 adults in the United States
}

\author{
*Jessica K Bone ${ }^{a}$, PhD \\ Hei Wan Maka, PhD \\ Jill K Sonke ${ }^{b}, \mathrm{PhD}$ \\ Meg E Fluhartya , PhD \\ Jenny Lee ${ }^{b}, \mathrm{MA}$ \\ Anthony J. Kolenic ${ }^{b}$, PhD \\ Heidi Radunovichc, PhD \\ Randy Cohen ${ }^{\text {, }}$ BA \\ Daisy Fancourt ${ }^{\mathrm{a}}$, PhD
}

a Research Department of Behavioural Science and Health, Institute of Epidemiology \& Health, University College London, London, UK

b Center for Arts in Medicine, University of Florida, Gainesville, Florida, US

c Family, Youth and Community Sciences, University of Florida, Gainesville, Florida, US

d Americans for the Arts, Washington DC, US

\section{*Corresponding author}

Address: Research Department of Behavioural Science and Health, 1-19 Torrington Place, London WC1E 7HB, UK

Email: jessica.bone@ucl.ac.uk 


\begin{abstract}
Arts engagement is a health-related behavior that may be influenced by social inequalities. While the COVID-19 pandemic provided new opportunities for some people to engage in the arts, it might have created barriers for others. We aimed to examine whether there was social patterning in home-based arts engagement during the pandemic in the United States (US), and whether predictors of engagement differed according to the type of arts activity. We included 4,731 adults who participated in the US COVID-19 Social Study between April and July 2020. Three types of home-based arts engagement were considered: reading for pleasure, arts or crafts activities, and digital arts activities. Using logistic regression models, we tested cross-sectional associations between a broad range of demographic, socioeconomic, psychosocial, and health-related factors as well as adverse events and worries during lockdown and each type of arts engagement. The factors most strongly associated with all three types of arts engagement were social support, social network size, age, race/ethnicity, keyworker status, and experiencing physical or psychological abuse during the pandemic. However, most socioeconomic and health-related factors were not associated with arts engagement, including household income and mental and physical health problems. Overall, our findings indicate that the social gradient in arts engagement was reduced in the first four months of the COVID-19 pandemic in the US. Given the health benefits of arts engagement, the potential diversification of arts audiences during the pandemic is promising for both population-level health and wellbeing and the future of the arts and cultural sector.
\end{abstract}

\title{
Keywords
}

arts engagement; cultural engagement; lockdown; stay-at-home orders; social gradient; health; wellbeing; US 
This manuscript is a preprint and has not been peer reviewed.

\section{Introduction}

There is extensive pre-pandemic evidence that arts engagement has beneficial impacts on mental and physical health (Fancourt \& Finn, 2019), and these benefits appear to have endured throughout the current coronavirus (COVID-19) pandemic. Spending more time on hobbies such as painting, writing, and other creative activities was associated with reductions in symptoms of depression and anxiety and improvements in life satisfaction during the first lockdown in the UK (Bu et al., 2021). People have reported using creative activities and consuming media to cope during lockdown, and arts activities helped frontline health and social care professionals to cope with challenges of the pandemic, supporting their mental health (Aughterson et al., 2021; Wright et al., 2021). Given the number of people experiencing distress, depression, anxiety, and loneliness during the COVID-19 pandemic (Bu et al., 2020; Holman et al., 2020), arts engagement is an important health behavior for improving the mental health and wellbeing of the population during the pandemic.

Despite unprecedented challenges for the arts and cultural sector during the COVID-19 pandemic, including the closure of community venues (Guibert \& Hyde, 2021), home-based arts engagement may have increased when people were directed to stay at home. In the US, there were large increases in online sales of arts and crafts products at the start of the pandemic (Choi et al., 2020). In the UK, nearly a quarter of 19,000 adults reported increases in their arts engagement during lockdown (Mak et al., 2021). These increases in arts engagement were likely due to a range of factors, including increased leisure time; using the arts to cope with boredom, stress, or health problems; using the arts to connect with others socially; and the increased availability and promotion of virtual resources, including online groups (e.g., choirs, book groups), streamed performances (e.g., plays, concerts), and digital arts activities (e.g., virtual museum tours).

However, while the pandemic provided new opportunities for some people to engage in the arts, it might have created barriers for others. In the UK, the social gradient in arts engagements was maintained; older adults, those with less education, and males had lower levels of arts engagement both before and during the pandemic (Mak et al., 2021). Despite this, those who had traditionally been excluded from the arts, such as people of ethnic minorities and those with health conditions, had similar levels of home-based arts engagement compared to others in the pandemic (Mak et al., 2021). However, a parallel study did not find evidence for changes in arts audiences in the UK, suggesting that pre-existing inequalities in arts engagement were maintained (Feder et al., 2021). Although the rapid increase in digital arts activities might reach new audiences, it may also have excluded individuals without access to reliable internet and those unable to use digital technology.

It remains unclear whether findings from the UK and Europe will generalize to the US. Pre-pandemic surveys have shown that, similar to the UK and Europe, arts engagement in the US differs according to socioeconomic position, education, and income (Bone et al., 2021; Stallings \& Mauldin, 2016). These factors mirror those that contribute to the inequalities in access to health care and health and social outcomes. However, the different social, cultural, and demographic context of the US, coupled with inconsistent, decentralized COVID-19 restrictions across states, could mean that the predictors and patterns of arts engagement during the pandemic differed to other countries. Additionally, different job subsidy structures could also have had distinct impacts on arts workers, affecting the provision of the arts during the pandemic. 
Therefore, in this study, we examined whether there was social patterning in home-based arts engagement during the pandemic in the US, and whether predictors of engagement differed according to the type of arts activity. We analyzed data from a large sample recruited during the first four months of stay-at-home directives. Three types of arts engagement were considered: reading for pleasure, arts or crafts activities, and digital arts activities. Understanding who is engaging in the arts in the US has significant policy implications, particularly after $96 \%$ of arts events were cancelled in the first five months of the pandemic (Americans for the Arts, 2021). Our findings should (a) reveal whether the usual predictors of arts engagement continued to affect audience profiles, or whether barriers to engagement were reduced for certain demographic groups in the US; (b) identify people who may be at risk of being excluded from the arts during a national crisis, which is important given the link between arts engagement and wellbeing; and (c) provide direction for arts organizations (Radermecker, 2021).

\section{Methods}

\section{Sample}

The study was a US extension of the UK COVID-19 Social Study run by University College London; a panel study collecting data weekly during the pandemic (https://github.com/UCLBSH/CSSUserGuide). The COVID-19 Social Study did not recruit a random sample and is thus not representative of the US population. However, it does contain a heterogeneous sample that was recruited using a snowballing approach with a focus on reaching diverse populations. National social, health, and arts organizations and networks shared the study invitation through their email lists and social media. We included 4,731 participants who participated between $6^{\text {th }}$ April and $23^{\text {rd }}$ July 2020 (see Supplement).

The study was approved by the UCL Research Ethics Committee (12467/005) and the University of Florida Institutional Review Board (IRB202000785). All participants gave informed consent.

\section{Measures}

\section{Arts engagement}

We measured arts engagement with three questions at one wave completed by participants between $6^{\text {th }}$ April and $23^{\text {rd }}$ July 2020 . These were part of a series of questions following a time diary approach, in which participants were asked to focus on the last weekday and consider how much time they spent on a range of activities. We chose weekdays to remove variation in responses due to whether participants took part on weekends. Participants were asked how long they had spent engaging in 1) reading for pleasure, 2) a home-based arts or crafts activity (e.g., painting, creative writing, sewing, playing music, etc.), and 3) digital arts activities (e.g., streaming a concert, virtual tour of a museum, etc.) on the last weekday. Given the low frequency of arts engagement, we created binary variables indicating whether participants had spent time on each of the activities (yes, no). 
This manuscript is a preprint and has not been peer reviewed.

\section{Predictors}

We measured a range of demographic, socioeconomic, psychosocial, and health-related factors as well as adverse events and worries during lockdown. Demographic factors included age (18-29, 30-59, $\geq 60$ years), gender (male, female), race/ethnicity (White, Black/African American, Asian/Asian American, Mixed Race, Other [including Latino/Hispanic, Chinese/Chinese American, Middle Eastern/Middle Eastern American, Native/American Indian/Alaska Native]), marital status (never married, divorced/widowed, in a relationship/married living apart, in a relationship/married cohabiting), living arrangement (alone, not alone but no children, not alone with children), and urbanicity of home area (rural, city/town).

Socioeconomic factors were employment status (unemployed, employed), highest level of education (high school or less, some college, undergraduate degree, postgraduate/professional degree), and household income $(<\$ 75,000, \geq \$ 75,000$ household income per annum). Binary variables (yes, no) measured household overcrowding ( $>1$ person per room, excluding the bathrooms and kitchen), homeowner status, and keyworker status (e.g., health or social care worker, teacher or childcare worker still travelling to work, transport worker, key public services).

We included three psychosocial and two health-related measures. Social support was measured with an adapted version of the 6-item Perceived Social Support Questionnaire. Higher scores indicate more perceived social support (range 6-30; Lin et al., 2019). Large social network was indicated by the number of friends $(<3, \geq 3)$. Loneliness was measured using the 3 -item Revised UCLA Loneliness Scale, with an additional item asking how often respondents felt lonely. Higher scores indicate greater loneliness (range 4-12; Russell et al., 1980). Participants were asked if they had been clinically diagnosed with a mental health condition (yes, no) or a physical condition/disability (yes, no).

Finally, we considered whether six adverse events were experienced or were a source of worry during the pandemic (yes, no): 1) COVID-19 diagnosis; 2) physical/psychological abuse (physically harmed/hurt, bullied, controlled, intimidated, or psychologically hurt by someone else); 3) financial difficulties (unable to pay bills/rent/mortgage or had a major cut in household income); 4) lost work; 5) difficulties accessing food; 6) difficulties accessing medication.

\section{Statistical analysis}

We used logistic regression to investigate the cross-sectional associations between predictors and each type of arts engagement (reading for pleasure, arts or crafts activities, digital arts activities) separately. All predictors were included in the model simultaneously.

We weighted the final analytical sample to match the characteristics of the non-institutionalized US population aged 18 and over by weighting according to age, gender, race/ethnicity, and education, obtained from the US Census Bureau (US Census Bureau, 2021), using the Stata package ebalance (Hainmueller \& Xu, 2013). To remove extreme variation, weights were trimmed to a maximum of the median plus six times the interquartile range, and then adjusted so that the total summed to the number of participants (Chowdhury et al., 2007; Potter \& Zheng, 2015). For comparison, unweighted analyses are presented in Table S1. All analyses were performed using Stata 16 (StataCorp, 2019). 


\section{Results}

Before weighting, $83 \%$ of the sample were female, $86 \%$ were of White race/ethnicity, and $47 \%$ had postgraduate/professional qualifications (Table 1). After weighting, $58 \%$ of the sample were female, $80 \%$ were of White race/ethnicity, and $18 \%$ had postgraduate/professional qualifications. Of this sample, $65 \%$ reported reading for pleasure on the last weekday, $42 \%$ did a home-based arts or crafts activity, and $20 \%$ did a digital arts activity.

[Table 1 here]

\section{Reading for pleasure}

The odds of reading for pleasure were higher for older participants and for those with increasing levels of education (Table 2). Participants who were in a relationship but living apart also had higher odds of reading for pleasure than those who were single. Additionally, participants reporting higher levels of social support had higher odds of reading for pleasure. There was no evidence that any other predictors were associated with reading for pleasure.

\section{Home-based arts or crafts}

Females had higher odds of engaging in home-based arts or crafts activities than males (Table 2). There was also evidence that participants of all races/ethnicities except Asian/Asian American were more likely to do arts or crafts activities than White participants. Those living with other adults and children were also more likely to do arts or crafts than those living alone. Additionally, individuals with more social support and a larger social network had higher odds of doing arts or crafts activities, as did those who had been physically or psychologically abused. However, keyworkers had lower odds of engaging in arts or crafts activities than non-keyworkers.

\section{Digital arts activities}

Compared to the youngest participants, those aged 60 and over had higher odds of engaging in digital arts activities (Table 2). There was also evidence that participants of all races/ethnicities except Mixed Race had higher odds of engaging in digital arts activities than White participants. As for home-based arts or crafts, individuals with more social support and a larger social network had higher odds of doing digital arts activities, as did those who had been physically or psychologically abused, and keyworkers had lower odds of engaging in digital arts activities.

[Table 2 here]

\section{Discussion}

In this study, a range of factors were independently associated with home-based arts engagement during the first four months of the pandemic. Around two thirds of participants reported reading for pleasure, over one third did a home-based arts or crafts activity, and one fifth did a digital arts activity on the last weekday. The strongest predictors of arts engagement were social support, social network size, age, race/ethnicity, keyworker status, and experiencing physical or psychological abuse during the pandemic. Other factors were only associated with one type of arts engagement. For example, 
females and those living with children were more likely to do arts or crafts, individuals in a relationship but living apart were more likely to read for pleasure, and higher levels of education were associated with more reading for pleasure, but not engaging in arts or crafts or digital arts activities. Most socioeconomic and health-related factors were not associated with arts engagement, including employment status, household income and overcrowding, loneliness, mental and physical health problems, having COVID-19, and other adverse events and worries experienced during the pandemic.

In comparison to before the pandemic, some predictors of arts engagement appear to have remained consistent. For example, higher levels of perceived social support and larger social networks were most consistently associated with arts engagement, as in pre-pandemic studies of home-based arts engagement (Fancourt et al., 2020) and cultural engagement more broadly (Fancourt \& Baxter, 2020). This may be because individuals with more supportive relationships receive more encouragement to participate in the arts, both in the form of material resources (e.g., paying for lessons or providing tangible resources such as books) and non-material resources (e.g., emotional support, awareness of online arts activities). This association may be bidirectional, as engagement could provide opportunities to connect with others and build a sense of community (Fancourt et al., 2021). We also found that females were more likely to do arts or crafts, which is in line with previous evidence before and during the pandemic (Bone et al., 2021; Mak et al., 2020, 2021; Stallings \& Mauldin, 2016).

However, some predictors of arts engagement in the US may have changed during the pandemic. Although higher levels of education were associated with reading for pleasure, education was not associated with doing arts or crafts or digital arts activities. This differs from pre-pandemic evidence that education is one of the strongest predictors of all forms of arts engagement (Bone et al., 2021; Stallings \& Mauldin, 2016). It is possible that the increased availability of digital arts activities created new opportunities for people from varied backgrounds to become aware of the arts during the pandemic. Additionally, the closure of museums, theatres, and arts venues could have predominantly impacted individuals with higher levels of education (Suarez-Fernandez et al., 2020).

Similarly, socioeconomic position is usually a strong predictor of broad arts engagement in the US (Bone et al., 2021; Stallings \& Mauldin, 2016), but we found no evidence that income was associated with home-based engagement during the pandemic, as shown in the UK during the pandemic (Mak et al., 2021). The lack of association with doing digital arts activities is particularly surprising given the link between household income and access to electronic devices and a stable internet connection. However, it could be because reading for pleasure, home-based arts or crafts, and digital arts activities are often affordable and easily accessible. In contrast, in-person cultural events can be expensive and require attendance at specific venues with associated transport costs.

Associations between race/ethnicity and arts engagement may also have changed in the pandemic. We found that reading for pleasure did not differ by race/ethnicity, but individuals who were not of White race/ethnicity were more likely to do arts or crafts and digital arts activities than people of White race/ethnicity. This may be due to the shift in location of arts activities from physical spaces rooted in white supremacy (e.g., art museums with colonial histories and racist legacies), which create a foundational barrier for Black, Indigenous, and other people of color (BIPOC groups), to digital interfaces that can be accessed from any location. 
Also in line with findings from the UK (Mak et al., 2021), arts engagement did not differ for individuals with or without physical or mental health conditions. In contrast, pre-pandemic studies of communitybased activities found that people with worse health and wellbeing were less likely to engage (Fancourt \& Baxter, 2020; Steptoe \& Fancourt, 2019). In addition to the greater accessibility and availability of home-based arts activities, individuals with existing health problems may have been more likely to use the arts to manage symptoms and cope with stress during the pandemic (Fancourt et al., 2019; Mak et al., 2021). Further, digital arts provision may have removed physical barriers relating to access. People who experienced physical or psychological abuse were more likely to engage in arts or crafts and digital arts activities, which might also be because these individuals were using the arts to cope and regulate their emotional responses to adverse events (Mak et al., 2021; Wright et al., 2021). The contrasting associations of social support and experiencing abuse with home-based arts engagement are particularly interesting and should be explored in future research.

Some of our findings may be a direct result of the pandemic and restrictions. Keyworkers, who continued travelling to work throughout the pandemic and may have faced increased working hours, stress, and burnout (Ruiz \& Gibson, 2020), were less likely to do arts or crafts and digital arts activities. They may not have benefitted from the increases in leisure time experienced by others and instead had a decreased work-life balance, making them less able to engage in arts activities. This is concerning given that this group may have particularly benefitted from the arts as a coping strategy; enabling keyworkers to engage in arts activities during future health emergencies should be a priority. Additionally, individuals living with children were more likely to do arts or crafts activities, which might have offered opportunities for parents to engage and bond with their children, as well as preventing boredom (Choi et al., 2020). Finally, older adults were more likely to read and engage in digital arts, which could be a result of the stricter stay-at-home directives for vulnerable older adults, with additional time at home providing increased opportunities and motivations to engage.

This study has several strengths, including the large sample recruited early in the pandemic. It was one of the first studies to examine arts engagement in the US during the pandemic. A wide range of sociodemographic information was collected, allowing comparison to similar data from the UK (Mak et al., 2021). However, the COVID-19 Social Study sample was not random and may have been biased towards individuals who were more engaged in the arts as participants were recruited with the help of Americans for the Arts. Whilst our data was weighted according to age, gender, race/ethnicity, and education distributions in the US population (US Census Bureau, 2021), we cannot rule out biases due to omitting other factors associated with survey participation in the weighting process. Given that the survey was completed online, our sample may exclude people who do not have digital access or comfort with digital interfaces.

Additionally, we excluded participants who did not identify as male or female to match our data to available population statistics. As the US Census Bureau only gives people the option to report their gender as male or female (US Census Bureau, 2021), the proportion of people in the US who do not identify as male or female is unknown, and we could not include these individuals in our sample weighting. Our weighted sample is thus not representative of all individuals in the US. We recognize that gender is not a binary construct and changes to the measures used by the US Census Bureau are needed. We also used an overly simple race/ethnicity variable due to small numbers in non-White groups, which conflates experiences across diverse racial/ethnic groups. Future research must include 
more diverse samples and collect detailed data on race/ethnicity. Furthermore, our analysis was based on cross-sectional data, meaning causality cannot be established. It is likely that some associations were bidirectional. As data collection began after the onset of the pandemic, we were not able to compare arts engagement during the pandemic to pre-pandemic behavior. Future research could investigate longer-term changes in arts engagement to better understand the changing characteristics of audiences in the US and whether differences are sustained post-pandemic.

Overall, our findings indicate that the social gradient in arts engagement was reduced in the first four months of the COVID-19 pandemic in the US. Additionally, individuals who were not of White race/ethnicity were more likely to engage in some arts activities. Our findings are generally in line with evidence from the UK, which also showed a narrowing of inequalities in arts engagement in the early months of the pandemic when individuals were restricted just to at-home engagement (Feder et al., 2021; Mak et al., 2021). Our findings contradict previous evidence that digital engagement replicates, or even enlarges, existing inequalities in community-based cultural engagement (Weingartner, 2020). It is likely that the pandemic was an exceptional situation, with many barriers to accessing the arts removed, enabling engagement for people from a wide range of backgrounds. Given the numerous health benefits of arts engagement (Fancourt \& Finn, 2019), the potential diversification of arts audiences during the pandemic is promising for both population-level health and wellbeing and the future of the arts sector. Additionally, increasing arts engagement in historically excluded groups could counteract inequalities in the impacts of the COVID-19 pandemic (Dorn et al., 2020). Future research should explore whether arts audiences changed further throughout the pandemic and how arts programming can be more responsive to community needs. Subsequent studies should also explore the specific conditions needed for people to continue participating following the pandemic and conditions needed to reduce barriers to arts participation that have persisted, particularly for BIPOC groups, in the US. This will require efforts to recruit more diverse and representative samples.

\section{Declarations}

Conflict of interest

No authors report any conflicts of interest.

\section{Acknowledgements}

The researchers are grateful for the support of Americans for the Arts and members of the University of Florida Center for Arts in Medicine Interdisciplinary Research Lab with their recruitment efforts. We also gratefully acknowledge the contribution of the COVID-19 Social Study participants.

\section{Funding}

This Covid-19 Social Study was funded by the Nuffield Foundation (WEL/FR-000022583), but the views expressed are those of the authors and not necessarily the Foundation. The study was also supported by the MARCH Mental Health Network funded by the Cross-Disciplinary Mental Health Network Plus initiative supported by UK Research and Innovation (ES/S002588/1), and by the Wellcome Trust (221400/Z/20/Z). The EpiArts Lab, a National Endowment for the Arts Research Lab at the University of Florida, is supported in part by an award from the National Endowment for the Arts (1862896-38- 
C-20). The opinions expressed are those of the authors and do not represent the views of the National Endowment for the Arts Office of Research \& Analysis or the National Endowment for the Arts. The National Endowment for the Arts does not guarantee the accuracy or completeness of the information included in this material and is not responsible for any consequences of its use. The EpiArts Lab is also supported by the University of Florida, the Pabst Steinmetz Foundation, and Bloomberg Philanthropies. DF is supported by the Wellcome Trust (205407/Z/16/Z). The funders had no final role in the study design; in the collection, analysis, and interpretation of data; in the writing of the report; or in the decision to submit the paper for publication. All researchers listed as authors are independent from the funders and all final decisions about the research were taken by the investigators and were unrestricted.

\section{Author contributions}

$J K S, J L, A J K, H R, R C$, and DF were responsible for the study protocol development and participant recruitment. JKB, HWM, DF and JKS designed the analyses in this manuscript. JKB conducted the analysis and JKB and HWM drafted the manuscript. JKB, HWM, JKS, MEF, JL, AJK, HR, RC, and DF contributed to the writing, made critical revisions, and approved the final manuscript. 
This manuscript is a preprint and has not been peer reviewed.

\section{References}

Americans for the Arts. (2021). The economic impact of coronavirus on the arts and culture sector. https://www.americansforthearts.org/by-topic/disaster-preparedness/the-economic-impact-of-coronaviruson-the-arts-and-culture-sector

Aughterson, H., McKinlay, A. R., Fancourt, D., \& Burton, A. (2021). Psychosocial impact on frontline health and social care professionals in the UK during the COVID-19 pandemic: A qualitative interview study. BMJ Open, 11(2), 110. https://doi.org/10.1136/bmjopen-2020-047353

Bone, J. K., Bu, F., Fluharty, M. E., Paul, E., Sonke, J. E., \& Fancourt, D. (2021). Who engages in the arts in the United States? A comparison of several types of engagement using data from the General Social Survey. BMC Public Health, 21, 1349. https://doi.org/10.1186/s12889-021-11263-0

Bu, F., Steptoe, A., \& Fancourt, D. (2020). Who is lonely in lockdown? Cross-cohort analyses of predictors of loneliness before and during the COVID-19 pandemic. Public Health, 186, 31-34. https://doi.org/10.1016/j.puhe.2020.06.036

Bu, F., Steptoe, A., Mak, H. W., \& Fancourt, D. (2021). Time use and mental health in UK adults during an 11-week COVID-19 lockdown: a panel analysis. The British Journal of Psychiatry, 219(4), 551-556. https://doi.org/10.1192/bjp.2021.44

Choi, M., Tessler, H., \& Kao, G. (2020). Arts and crafts as an educational strategy and coping mechanism for Republic of Korea and United States parents during the COVID-19 pandemic. International Review of Education, 66(5-6), 715-735. https://doi.org/10.1007/s11159-020-09865-8

Chowdhury, S., Khare, M., \& Wolter, K. (2007). Weight trimming in the national immunization survey. In Proceedings of the Joint Statistical Meetings, Section on Survey Research Methods (pp. 2651-2658). American Statistical Association.

Dorn, A. van, Cooney, R. E., \& Sabin, M. L. (2020). COVID-19 exacerbating inequalities in the US. Lancet, 395, 12431244.

Fancourt, D., Aughterson, H., Finn, S., Walker, E., \& Steptoe, A. (2021). How leisure activities affect health: a narrative review and multi-level theoretical framework of mechanisms of action. The Lancet Psychiatry, 8(4), 329-339. https://doi.org/10.1016/ S2215-0366(20)30384-9

Fancourt, D., \& Baxter, L. (2020). Differential participation in community cultural activities amongst those with poor mental health: Analyses of the UK Taking Part Survey. Social Science and Medicine, 261(March), 113221. https://doi.org/10.1016/j.socscimed.2020.113221

Fancourt, D., Baxter, L., \& Lorencatto, F. (2020). Barriers and enablers to engagement in participatory arts activities amongst individuals with depression and anxiety: Quantitative analyses using a behaviour change framework. BMC Public Health, 20(1), 1-12. https://doi.org/10.1186/s12889-020-8337-1

Fancourt, D., \& Finn, S. (2019). What is the evidence on the role of the arts in improving health and well-being? A scoping review. https://europepmc.org/article/NBK/nbk553773

Fancourt, D., Garnett, C., Spiro, N., West, R., \& Müllensiefen, D. (2019). How do artistic creative activities regulate our emotions? Validation of the Emotion Regulation Strategies for Artistic Creative Activities Scale (ERS-ACA). PLOS ONE, 14(2), 1-22. https://doi.org/10.1371/journal.pone.0211362

Feder, T., McAndrew, S., O'Brien, D., \& Taylor, M. (2021). Cultural Consumption and Covid-19: Evidence from the Taking Part and COVID-19 Cultural Participation Monitor surveys. SocArXiv.

Guibert, G., \& Hyde, I. (2021). ANALYSIS: COVID-19's Impacts on Arts and Culture. https://www.arts.gov/sites/default/files/COVID-Outlook-Week-of-1.4.2021-revised.pdf

Hainmueller, J., \& Xu, Y. (2013). Ebalance: A stata package for entropy balancing. Journal of Statistical Software, 54(7), 1-18. https://doi.org/10.18637/jss.v054.i07

Holman, E. A., Thompson, R. R., Garfin, D. R., \& Silver, R. C. (2020). The unfolding COVID-19 pandemic: A probabilitybased, nationally representative study of mental health in the United States. Science Advances, 6(42), 1-8. https://doi.org/10.1126/sciadv.abd5390 
This manuscript is a preprint and has not been peer reviewed.

Lin, M., Hirschfeld, G., \& Margraf, J. (2019). Brief form of the perceived social support questionnaire (F-SozU K-6): validation, norms, and cross-cultural measurement invariance in the USA, Germany, Russia, and China. Psychological Assessment, 31, 609-621. https://doi.org/10.1037/pas0000686

Mak, H. W., Coulter, R., \& Fancourt, D. (2020). Patterns of social inequality in arts and cultural participation: Findings from a nationally representative sample of adults living in the United Kingdom of Great Britain and Northern Ireland. Public Health Panorama, 6(1), 55-68.

Mak, H. W., Fluharty, M., \& Fancourt, D. (2021). Predictors and Impact of Arts Engagement During the COVID-19 Pandemic: Analyses of Data From 19,384 Adults in the COVID-19 Social Study. Frontiers in Psychology, 12. https://doi.org/10.3389/fpsyg.2021.626263

Potter, F., \& Zheng, Y. (2015). Methods and Issues in Trimming Extreme Weights in Sample Surveys. In Proceedings of the American Statistical Association, Section on Survey Research Methods (pp. 2707-2719). American Statistical Association.

Radermecker, A.-S. v. (2021). Art and culture in the COVID-19 era: for a consumer-oriented approach. SN Business \& Economics, 1(1), 1-14. https://doi.org/10.1007/s43546-020-00003-y

Ruiz, M. A., \& Gibson, C. A. M. (2020). Emotional impact of the COVID-19 pandemic on U.S. health care workers: A gathering storm. Psychological Trauma: Theory, Research, Practice, and Policy, 12, S153-S155. https://doi.org/10.1037/tra0000851

Russell, D., Peplau, L. A., \& Cutrona, C. E. (1980). The revised UCLA loneliness scale: concurrent and discriminant validity evidence. Journal of Personality and Social Psychology, 39, 472-480. https://doi.org/10.1037/00223514.39.3.472

Stallings, S. N., \& Mauldin, B. (2016). Public Engagement in the Arts: A Review of Recent Literature. https://www.lacountyarts.org/sites/default/files/pdfs/lacac_pubenglitrev.pdf

StataCorp. (2019). Stata Statistical Software: Release 16. StataCorp LP.

Steptoe, A., \& Fancourt, D. (2019). Leading a meaningful life at older ages and its relationship with social engagement, prosperity, health, biology, and time use. Proceedings of the National Academy of Sciences of the United States of America, 116(4), 1207-1212. https://doi.org/10.1073/pnas.1814723116

Suarez-Fernandez, S., Prieto-Rodriguez, J., \& Perez-Villadoniga, M. J. (2020). The changing role of education as we move from popular to highbrow culture. Journal of Cultural Economics, 44(2), 189-212. https://doi.org/10.1007/s10824-019-09355-2

US Census Bureau. (2021). US Population. https://www.census.gov/topics/population.html

Weingartner, S. (2020). Digital omnivores? How digital media reinforce social inequalities in cultural consumption. New Media and Society. https://doi.org/10.1177/1461444820957635

Wright, L., Fluharty, M. E., Steptoe, A., \& Fancourt, D. (2021). How did people cope during the COVID-19 pandemic? A Structural Topic Modelling Analysis of Free-Text Data from 11,000 UK Adults. MedRxiv. https://doi.org/10.1101/2021.08.13.21262002 
This manuscript is a preprint and has not been peer reviewed.

Tables and figures

Table 1. Sociodemographic characteristics of the sample and arts engagement on the last weekday.

\begin{tabular}{|c|c|c|}
\hline Sociodemographic characteristics & Unweighted & Weighted \\
\hline \multicolumn{3}{|l|}{ Age group } \\
\hline $18-29$ & $15 \%$ & $19 \%$ \\
\hline $30-59$ & $53 \%$ & $49 \%$ \\
\hline $60+$ & $32 \%$ & $32 \%$ \\
\hline \multicolumn{3}{|l|}{ Gender } \\
\hline Male & $17 \%$ & $42 \%$ \\
\hline Female & $83 \%$ & $58 \%$ \\
\hline \multicolumn{3}{|l|}{ Race/ethnicity } \\
\hline White & $86 \%$ & $80 \%$ \\
\hline Black/African American & $3 \%$ & $10 \%$ \\
\hline Asian/Asian American & $2 \%$ & $5 \%$ \\
\hline Mixed Race & $4 \%$ & $3 \%$ \\
\hline Other & $5 \%$ & $2 \%$ \\
\hline \multicolumn{3}{|l|}{ Marital status } \\
\hline Single, never married & $20 \%$ & $26 \%$ \\
\hline Single, divorced or widowed & $16 \%$ & $17 \%$ \\
\hline In a relationship/married, living apart & $6 \%$ & $7 \%$ \\
\hline In a relationship/married, cohabiting & $57 \%$ & $51 \%$ \\
\hline \multicolumn{3}{|l|}{ Living arrangement } \\
\hline Alone & $22 \%$ & $20 \%$ \\
\hline Not alone, no child & $54 \%$ & $55 \%$ \\
\hline Not alone, with child & $24 \%$ & $25 \%$ \\
\hline \multicolumn{3}{|l|}{ Urbanicity of home area } \\
\hline Rural & $11 \%$ & $14 \%$ \\
\hline City/town & $89 \%$ & $86 \%$ \\
\hline \multicolumn{3}{|l|}{ Employment status } \\
\hline Unemployed & $35 \%$ & $45 \%$ \\
\hline Employed & $65 \%$ & $55 \%$ \\
\hline \multicolumn{3}{|l|}{ Education } \\
\hline High school or less & $3 \%$ & $15 \%$ \\
\hline Some college & $18 \%$ & $37 \%$ \\
\hline Undergraduate & $32 \%$ & $30 \%$ \\
\hline Postgraduate/professional & $47 \%$ & $18 \%$ \\
\hline High household income $(\$ 75,000+)$ & $55 \%$ & $44 \%$ \\
\hline Household overcrowded & $2 \%$ & $4 \%$ \\
\hline Homeowner & $63 \%$ & $59 \%$ \\
\hline Keyworker & $23 \%$ & $23 \%$ \\
\hline Social support (range 6-30) - mean (SD) & $23.25(5.81)$ & $22.04(6.42)$ \\
\hline Loneliness (range 4-12) - mean (SD) & $6.96(2.54)$ & $7.20(2.70)$ \\
\hline Large social network ( $3+$ friends) & $74 \%$ & $66 \%$ \\
\hline Mental health problem & $38 \%$ & $39 \%$ \\
\hline Physical health problem & $47 \%$ & $51 \%$ \\
\hline Had COVID-19 & $9 \%$ & $8 \%$ \\
\hline Physically/psychologically abused & $10 \%$ & $12 \%$ \\
\hline Financial difficulties & $17 \%$ & $21 \%$ \\
\hline Lost work & $9 \%$ & $11 \%$ \\
\hline Difficulties accessing food & $3 \%$ & $5 \%$ \\
\hline Difficulties accessing medication & $2 \%$ & $3 \%$ \\
\hline Worried about COVID-19 & $53 \%$ & $50 \%$ \\
\hline Worried about personal safety & $21 \%$ & $22 \%$ \\
\hline Worried about finances & $45 \%$ & $47 \%$ \\
\hline Worried about work & $24 \%$ & $23 \%$ \\
\hline Worried about food access & $16 \%$ & $16 \%$ \\
\hline Worried about medication access & $10 \%$ & $11 \%$ \\
\hline \multicolumn{3}{|l|}{ Arts engagement on last weekday } \\
\hline Read for pleasure & $67 \%$ & $65 \%$ \\
\hline Did home-based arts or crafts activity & $43 \%$ & $42 \%$ \\
\hline Did digital arts activity & $21 \%$ & $20 \%$ \\
\hline
\end{tabular}

Note. $\mathrm{N}=4,731$. Data were weighted by age, gender, race/ethnicity, and education. 
This manuscript is a preprint and has not been peer reviewed.

Table 2. Associations between predictors and each type of arts engagement.

\begin{tabular}{|c|c|c|c|c|c|c|}
\hline & \multicolumn{2}{|c|}{ Reading for pleasure } & \multicolumn{2}{|c|}{ Arts or crafts activities } & \multicolumn{2}{|c|}{ Digital arts activities } \\
\hline & OR $(95 \% \mathrm{Cl})$ & $\mathrm{p}$ & OR $(95 \% \mathrm{Cl})$ & $p$ & OR $(95 \% \mathrm{Cl})$ & $p$ \\
\hline \multicolumn{7}{|l|}{ Age group (vs 18-29) } \\
\hline $30-59$ & $1.45(1.04-2.02)$ & 0.029 & $0.84(0.60-1.17)$ & 0.290 & $1.47(0.94-2.30)$ & 0.090 \\
\hline $60+$ & $3.25(2.12-4.98)$ & $<0.001$ & $0.73(0.49-1.11)$ & 0.142 & $2.33(1.39-3.90)$ & 0.001 \\
\hline Female (vs male) & $0.95(0.75-1.19)$ & 0.633 & $1.77(1.42-2.21)$ & $<0.001$ & $0.78(0.59-1.03)$ & 0.076 \\
\hline \multicolumn{7}{|l|}{ Race/ethnicity (vs White) } \\
\hline Black/African American & $1.48(0.93-2.35)$ & 0.097 & $1.64(1.06-2.53)$ & 0.027 & $2.43(1.53-3.85)$ & $<0.001$ \\
\hline Asian/Asian American & $0.91(0.56-1.48)$ & 0.714 & $1.14(0.68-1.91)$ & 0.614 & $1.92(1.02-3.60)$ & 0.044 \\
\hline Mixed Race & $0.97(0.51-1.85)$ & 0.928 & $2.15(1.26-3.67)$ & 0.005 & $1.69(0.91-3.15)$ & 0.098 \\
\hline Other & $1.20(0.64-2.27)$ & 0.570 & $2.51(1.42-4.44)$ & 0.002 & $2.47(1.28-4.78)$ & 0.007 \\
\hline \multicolumn{7}{|l|}{ Marital status (vs single, never married) } \\
\hline Single, divorced or widowed & $1.10(0.75-1.61)$ & 0.637 & $1.16(0.79-1.68)$ & 0.453 & $0.96(0.61-1.50)$ & 0.843 \\
\hline In a relationship/married, living apart & $1.73(1.10-2.72)$ & 0.018 & $1.00(0.64-1.57)$ & 0.999 & $0.71(0.39-1.30)$ & 0.270 \\
\hline In a relationship/married, cohabiting & $0.93(0.66-1.29)$ & 0.651 & $1.07(0.76-1.49)$ & 0.703 & $0.76(0.50-1.15)$ & 0.195 \\
\hline \multicolumn{7}{|l|}{ Living arrangement (vs alone) } \\
\hline Not alone, no child & $1.03(0.73-1.46)$ & 0.858 & $1.08(0.78-1.51)$ & 0.635 & $1.11(0.74-1.68)$ & 0.607 \\
\hline Not alone, with child & $1.01(0.68-1.50)$ & 0.967 & $1.47(1.00-2.14)$ & 0.047 & $1.41(0.89-2.25)$ & 0.144 \\
\hline Lives in city/town (vs rural) & $1.06(0.76-1.46)$ & 0.741 & $0.95(0.69-1.31)$ & 0.767 & $1.04(0.73-1.49)$ & 0.834 \\
\hline Employed (vs unemployed) & $0.83(0.64-1.07)$ & 0.152 & $0.96(0.75-1.23)$ & 0.732 & $1.22(0.90-1.65)$ & 0.208 \\
\hline \multicolumn{7}{|l|}{ Education (vs high school or less) } \\
\hline Some college & $1.58(1.04-2.39)$ & 0.032 & $1.33(0.90-1.96)$ & 0.150 & $1.49(0.87-2.55)$ & 0.146 \\
\hline Undergraduate & $1.62(1.07-2.46)$ & 0.022 & $1.00(0.68-1.47)$ & 0.995 & $1.52(0.90-2.58)$ & 0.116 \\
\hline Postgraduate/professional & $1.70(1.11-2.60)$ & 0.015 & $0.94(0.63-1.40)$ & 0.758 & $1.58(0.93-2.69)$ & 0.091 \\
\hline High household income $(\$ 75,000+)$ & $1.22(0.96-1.55)$ & 0.110 & $0.84(0.66-1.06)$ & 0.142 & $1.18(0.88-1.58)$ & 0.274 \\
\hline Household overcrowded & $0.88(0.50-1.53)$ & 0.641 & $1.02(0.58-1.79)$ & 0.938 & $0.94(0.47-1.85)$ & 0.852 \\
\hline Homeowner & $1.20(0.93-1.55)$ & 0.163 & $1.10(0.86-1.40)$ & 0.445 & $1.09(0.81-1.45)$ & 0.566 \\
\hline Keyworker & $0.92(0.71-1.18)$ & 0.499 & $0.68(0.52-0.89)$ & 0.004 & $0.62(0.45-0.85)$ & 0.003 \\
\hline Social support & $1.03(1.01-1.05)$ & 0.012 & $1.03(1.00-1.05)$ & 0.039 & $1.04(1.01-1.07)$ & 0.009 \\
\hline Loneliness & $0.97(0.92-1.02)$ & 0.277 & $0.99(0.94-1.05)$ & 0.750 & $0.99(0.92-1.06)$ & 0.715 \\
\hline Large social network (3+ friends) & $1.21(0.94-1.56)$ & 0.140 & $1.32(1.03-1.69)$ & 0.031 & $1.43(1.07-1.91)$ & 0.016 \\
\hline Mental health problem & $0.94(0.74-1.19)$ & 0.617 & $0.96(0.76-1.20)$ & 0.692 & $0.95(0.72-1.27)$ & 0.748 \\
\hline Physical health problem & $0.88(0.70-1.12)$ & 0.297 & $0.87(0.69-1.09)$ & 0.221 & $0.83(0.63-1.10)$ & 0.198 \\
\hline Had COVID-19 & $1.26(0.86-1.83)$ & 0.234 & $0.82(0.57-1.17)$ & 0.270 & $1.38(0.89-2.13)$ & 0.150 \\
\hline Physically/psychologically abused & $1.24(0.85-1.79)$ & 0.261 & $1.47(1.04-2.09)$ & 0.030 & $2.41(1.64-3.55)$ & $<0.001$ \\
\hline Financial difficulties & $1.08(0.79-1.49)$ & 0.635 & $1.33(0.99-1.80)$ & 0.061 & $1.37(0.98-1.92)$ & 0.067 \\
\hline Lost work & $1.31(0.88-1.94)$ & 0.183 & $1.37(0.94-2.01)$ & 0.104 & $1.66(1.08-2.55)$ & 0.021 \\
\hline Difficulties accessing food & $1.62(0.84-3.14)$ & 0.153 & $0.91(0.46-1.78)$ & 0.780 & $1.08(0.52-2.26)$ & 0.837 \\
\hline Difficulties accessing medication & $1.21(0.52-2.77)$ & 0.660 & $0.92(0.45-1.90)$ & 0.830 & $0.65(0.29-1.45)$ & 0.290 \\
\hline Worried about COVID-19 & $0.92(0.73-1.16)$ & 0.477 & $1.06(0.85-1.32)$ & 0.582 & $1.17(0.89-1.52)$ & 0.259 \\
\hline Worried about personal safety & $1.02(0.77-1.36)$ & 0.888 & $1.12(0.85-1.47)$ & 0.413 & $1.02(0.73-1.42)$ & 0.900 \\
\hline Worried about finances & $1.28(1.00-1.65)$ & 0.053 & $1.12(0.88-1.42)$ & 0.358 & $0.97(0.72-1.30)$ & 0.840 \\
\hline Worried about work & $0.95(0.72-1.26)$ & 0.733 & $1.06(0.80-1.41)$ & 0.663 & $1.32(0.95-1.84)$ & 0.099 \\
\hline Worried about food access & $1.25(0.89-1.75)$ & 0.205 & $0.90(0.64-1.25)$ & 0.514 & $1.06(0.73-1.54)$ & 0.766 \\
\hline Worried about medication access & $0.92(0.61-1.40)$ & 0.713 & $1.39(0.93-2.08)$ & 0.108 & $1.13(0.72-1.77)$ & 0.603 \\
\hline
\end{tabular}

Note. $\mathrm{N}=4,731$. Data were weighted by age, gender, race/ethnicity, and education. Reference categories are shown in brackets. Bold font indicates significant results at $p<0.05$. 
This manuscript is a preprint and has not been peer reviewed.

\section{Supplementary Materials}

\section{Sample}

The study was launched as a US extension of the UK COVID-19 Social Study run by University College London; a longitudinal study that focuses on the psychological and social experiences of adults living during the COVID-19 pandemic (https://github.com/UCL-BSH/CSSUserGuide). The US extension used the same measures as the UK COVID19 Social Study and was delivered in partnership with the University of Florida Center for Arts in Medicine and Americans for the Arts, a non-profit organization whose primary focus is advancing the arts in the US (https://www.americansforthearts.org). In this study, we only included participants from the US extension of the COVID-19 Social Study. Data collection in the US began on $6^{\text {th }}$ April 2020, with participants initially completing a baseline survey, followed by weekly data collection for a maximum of 12 waves.

The COVID-19 Social Study did not include a random sample, but it does contain a heterogeneous sample recruited using a snowballing approach with a focus on reaching diverse populations. National social, health, and arts organizations and networks shared the study invitation through their email lists and social media. The study was approved by the UCL Research Ethics Committee (12467/005) and the University of Florida Institutional Review Board (IRB202000785). All participants gave informed consent. A full protocol for the primary study is available online at https://github.com/UCL-BSH/CSSUserGuide.

A total of 6,781 participants were recruited in the US. We selected our cross-sectional sample by including the first wave of data collected on arts engagement for each participant between $6^{\text {th }}$ April and $23^{\text {rd }}$ July 2020 , leaving a sample of 5,242 participants. We then further restricted the sample to participants who were: 18 and over ( $\mathrm{n}=2$ excluded); male or female (so that weights could be created; $n=61$ ); and had complete data on arts engagement and sociodemographic factors $(n=448)$. Although participants reported their gender as male, female, or other, we weighted analyses according to the statistics available from the US Census Bureau who still use a binary measure of gender (US Census Bureau, 2021), leading to the exclusion of participants reporting their gender as other. This resulted in a final analytical sample of 4,731 participants. 
This manuscript is a preprint and has not been peer reviewed.

Table S1. Associations between predictors and the odds of engaging in each type of arts engagement (unweighted).

\begin{tabular}{|c|c|c|c|c|c|c|}
\hline & \multicolumn{2}{|c|}{ Reading for pleasure } & \multicolumn{2}{|c|}{ Arts or crafts activities } & \multicolumn{2}{|c|}{ Digital arts activities } \\
\hline & OR $(95 \% \mathrm{Cl})$ & $\mathrm{p}$ & OR $(95 \% \mathrm{Cl})$ & $p$ & OR $(95 \% \mathrm{Cl})$ & $p$ \\
\hline \multicolumn{7}{|l|}{ Age group (vs 18-29) } \\
\hline $30-59$ & $1.65(1.35-2.01)$ & $<0.001$ & $1.07(0.88-1.30)$ & 0.513 & $1.44(1.11-1.86)$ & 0.006 \\
\hline $60+$ & $3.51(2.72-4.52)$ & $<0.001$ & $1.22(0.96-1.55)$ & 0.100 & $2.44(1.81-3.30)$ & $<0.001$ \\
\hline Female (vs male) & $0.98(0.82-1.17)$ & 0.837 & $1.98(1.66-2.35)$ & $<0.001$ & $0.97(0.80-1.19)$ & 0.794 \\
\hline \multicolumn{7}{|l|}{ Race/ethnicity (vs White) } \\
\hline Black/African American & $1.30(0.91-1.87)$ & 0.147 & $1.44(1.04-2.00)$ & 0.027 & $2.26(1.60-3.20)$ & $<0.001$ \\
\hline Asian/Asian American & $1.03(0.66-1.59)$ & 0.901 & $1.26(0.82-1.94)$ & 0.286 & $1.54(0.93-2.53)$ & 0.092 \\
\hline Mixed Race & $1.27(0.90-1.79)$ & 0.177 & $1.66(1.21-2.28)$ & 0.002 & $1.15(0.79-1.70)$ & 0.463 \\
\hline Other & $0.90(0.68-1.19)$ & 0.462 & $1.68(1.28-2.22)$ & $<0.001$ & $1.61(1.18-2.21)$ & 0.003 \\
\hline \multicolumn{7}{|l|}{ Marital status (vs single, never married) } \\
\hline Single, divorced or widowed & $0.85(0.67-1.07)$ & 0.164 & $0.91(0.73-1.13)$ & 0.410 & $0.94(0.72-1.22)$ & 0.627 \\
\hline In a relationship/married, living apart & $1.23(0.92-1.64)$ & 0.165 & $0.93(0.70-1.22)$ & 0.579 & $0.79(0.56-1.12)$ & 0.178 \\
\hline In a relationship/married, cohabiting & $0.82(0.67-1.01)$ & 0.066 & $0.97(0.79-1.18)$ & 0.760 & $0.80(0.62-1.02)$ & 0.075 \\
\hline \multicolumn{7}{|l|}{ Living arrangement (vs alone) } \\
\hline Not alone, no child & $1.11(0.90-1.37)$ & 0.343 & $1.09(0.90-1.33)$ & 0.380 & $1.10(0.87-1.40)$ & 0.440 \\
\hline Not alone, with child & $0.97(0.77-1.23)$ & 0.825 & $1.25(0.99-1.56)$ & 0.057 & $1.14(0.87-1.50)$ & 0.345 \\
\hline Lives in city/town (vs rural) & $0.92(0.75-1.13)$ & 0.423 & $0.86(0.71-1.04)$ & 0.118 & $0.97(0.77-1.22)$ & 0.781 \\
\hline Employed (vs unemployed) & $0.88(0.75-1.03)$ & 0.103 & $0.95(0.82-1.10)$ & 0.524 & $1.18(0.99-1.41)$ & 0.060 \\
\hline \multicolumn{7}{|l|}{ Education (vs high school or less) } \\
\hline Some college & $1.37(0.93-2.01)$ & 0.110 & $1.14(0.78-1.66)$ & 0.505 & $1.41(0.84-2.36)$ & 0.192 \\
\hline Undergrad & $1.48(1.01-2.16)$ & 0.043 & $0.97(0.67-1.41)$ & 0.888 & $1.44(0.87-2.39)$ & 0.161 \\
\hline Postgrad/professional & $1.55(1.06-2.27)$ & 0.023 & $0.92(0.64-1.34)$ & 0.680 & $1.47(0.88-2.44)$ & 0.140 \\
\hline High household income $(\$ 75,000+)$ & $1.04(0.89-1.21)$ & 0.615 & $0.80(0.70-0.92)$ & 0.002 & $1.11(0.93-1.31)$ & 0.241 \\
\hline Household overcrowded & $1.03(0.69-1.54)$ & 0.894 & $1.23(0.82-1.83)$ & 0.312 & $1.09(0.69-1.75)$ & 0.706 \\
\hline Homeowner & $1.19(1.02-1.38)$ & 0.026 & $1.10(0.95-1.27)$ & 0.200 & $1.00(0.84-1.19)$ & 0.969 \\
\hline Keyworker & $0.79(0.68-0.92)$ & 0.003 & $0.78(0.67-0.91)$ & 0.001 & $0.75(0.62-0.91)$ & 0.003 \\
\hline Social support & $1.02(1.01-1.04)$ & 0.005 & $1.03(1.01-1.04)$ & $<0.001$ & $1.02(1.01-1.04)$ & 0.004 \\
\hline Loneliness & $0.97(0.94-1.00)$ & 0.081 & $1.01(0.98-1.04)$ & 0.622 & $1.02(0.98-1.06)$ & 0.257 \\
\hline Large social network (3+ friends) & $1.30(1.11-1.52)$ & 0.001 & $1.23(1.05-1.43)$ & 0.008 & $1.34(1.11-1.62)$ & 0.003 \\
\hline Mental health problem & $0.96(0.84-1.10)$ & 0.570 & $0.99(0.87-1.13)$ & 0.889 & $0.81(0.69-0.95)$ & 0.011 \\
\hline Physical health problem & $0.93(0.81-1.06)$ & 0.280 & $1.00(0.88-1.14)$ & 0.997 & $0.98(0.84-1.15)$ & 0.834 \\
\hline Had COVID-19 & $1.31(1.04-1.65)$ & 0.023 & $1.16(0.94-1.44)$ & 0.161 & $1.10(0.86-1.42)$ & 0.440 \\
\hline Physically/psychologically abused & $1.01(0.81-1.26)$ & 0.910 & $1.28(1.04-1.58)$ & 0.019 & $1.40(1.10-1.78)$ & 0.006 \\
\hline Financial difficulties & $0.96(0.80-1.16)$ & 0.706 & $1.36(1.14-1.63)$ & 0.001 & $1.28(1.04-1.57)$ & 0.020 \\
\hline Lost work & $1.04(0.82-1.31)$ & 0.739 & $1.09(0.88-1.36)$ & 0.425 & $1.47(1.15-1.88)$ & 0.002 \\
\hline Difficulties accessing food & $1.16(0.77-1.75)$ & 0.480 & $1.04(0.70-1.54)$ & 0.853 & $0.71(0.43-1.15)$ & 0.166 \\
\hline Difficulties accessing medication & $1.28(0.80-2.05)$ & 0.294 & $1.06(0.69-1.65)$ & 0.789 & $1.16(0.69-1.94)$ & 0.572 \\
\hline Worried about COVID-19 & $0.91(0.79-1.04)$ & 0.162 & $1.04(0.92-1.19)$ & 0.511 & $1.01(0.86-1.18)$ & 0.897 \\
\hline Worried about personal safety & $0.96(0.81-1.13)$ & 0.616 & $1.15(0.98-1.35)$ & 0.077 & $1.06(0.87-1.28)$ & 0.566 \\
\hline Worried about finances & $1.18(1.02-1.37)$ & 0.024 & $1.07(0.93-1.23)$ & 0.329 & $1.08(0.91-1.27)$ & 0.390 \\
\hline Worried about work & $0.94(0.80-1.11)$ & 0.455 & $1.04(0.89-1.22)$ & 0.601 & $1.21(1.00-1.46)$ & 0.051 \\
\hline Worried about food access & $0.99(0.82-1.20)$ & 0.944 & $1.08(0.90-1.29)$ & 0.428 & $1.24(1.00-1.53)$ & 0.049 \\
\hline Worried about medication access & $1.00(0.79-1.27)$ & 0.998 & $1.27(1.01-1.60)$ & 0.040 & $1.06(0.80-1.40)$ & 0.680 \\
\hline
\end{tabular}

Note. $\mathrm{N}=4,731$. Reference categories are shown in brackets. Bold font indicates significant results at $\mathrm{p}<0.05$. 